\title{
Hydroxychloroquine and ritonavir for COVID-19 infection: a possible synergic toxicity for retinal pigmented epithelium
}

\author{
Mario R. Romano ${ }^{1} \cdot$ Raffaele Raimondi $^{1}$ (D) - Alessio Montericcio ${ }^{1} \cdot$ Davide Allegrini $^{1}$ \\ Received: 17 April 2020 / Revised: 17 April 2020 / Accepted: 26 April 2020 / Published online: 8 May 2020 \\ (C) Springer-Verlag GmbH Germany, part of Springer Nature 2020
}

Dear Editor,

The World Health Organization (WHO) launched the SOLIDARITY trial on 20 March which includes already approved drugs for other diseases repurposed to treat COVID-19 [1]. Mechanisms of action of these drugs are already known as well as their toxicity. We highlight the potential risks of combining drugs with established macular toxicity that are hydroxychloroquine and ritonavir, as actually performed in our Lombardia region, the heart of COVID-19 Italian breakout [2].

Chloroquine and his hydroxylated analog hydroxychloroquine were initially used as antimalarial agents and then extended to rheumatic disorders [3]. Long-term toxicity of chloroquine and hydroxychloroquine has been widely demonstrated, and recommended protocols as the one proposed by the American Academy of Ophthalmology (AAO) are followed worldwide; acute toxicity has also been reported after treatments at standard dosage after just 2 months $[4,5]$. Risk factors include a preexisting maculopathy, renal disease, and tamoxifen use. Tamoxifen use increased the risk of toxicity by 5 -folds; this drug has a reported retinal toxicity on its own, and therefore, a synergic toxicity has been hypothesized [4].

Ritonavir is an antiviral drug used in the highly active antiretroviral therapy (HAART) against HIV; it acts as a protease inhibitor and also blocks cytochrome P450; thus, it is given in combination with other antivirals, like lopinavir, to boost their effect. Its toxicity has never been investigated in depth, but since the first experimental studies, hypertrophy of the retinal pigmented epithelium (RPE) was reported. Multiple case

Raffaele Raimondi raffor9@gmail.com

1 Humanitas University, Via Rita Levi Montalcini 4, Pieve Emanuele, Milan, Italy reports described toxicity for the RPE with a bull's eye pattern that resembles the one observed with hydroxychloroquine [6].

Therefore, we alert for a possible synergic toxicity for the retina, just like what happens with hydroxychloroquine and tamoxifen and postulate the need for an ophthalmologic follow-up program for these patients.

\section{Compliance with ethical standards}

Conflicts of interest The authors declare that they have no conflict of interest.

\section{References}

1. Kupferschmidt K, Cohen J (2020) Race to find COVID-19 treatments accelerates. Science https://doi.org/10.1126/science.367. 6485.1412

2. Italian society of infective and tropical disease. (SIMEU) (2020) COVID-19 therapeutic protocol in Lombardia region, Italy. 1-15

3. Costedoat-Chalumeau N, Dunogué B, Leroux G et al (2015) A critical review of the effects of hydroxychloroquine and chloroquine on the eye. Clin Rev Allergy Immunol 49:317-326. https://doi.org/10. 1007/s12016-015-8469-8

4. Marmor MF, Kellner U, Lai TYY et al (2016) Recommendations on screening for chloroquine and hydroxychloroquine retinopathy (2016 revision). Ophthalmology 123:1386-1394. https://doi.org/10. 1016/j.ophtha.2016.01.058

5. Pasaoglu Isil OFE (2019) Macular toxicity after short-term hydroxychloroquine therapy. Indian J Ophthalmol

6. Louie AK, Jones HN (2019) Case report: retinal toxicity secondary to ritonavir. Optom Vis Sci 96:376-381. https://doi.org/10.1097/ OPX.0000000000001368

Publisher's note Springer Nature remains neutral with regard to jurisdictional claims in published maps and institutional affiliations. 